\title{
ASSOCIAÇÃO ENTRE FORÇA MUSCULAR E SENSIBILIDADE PLANTAR EM PACIENTES DIABETICOS: UM ESTUDO TRANSVERSAL
}

\section{Tiago Camillo Veras \\ Lélio Russell de Moura Rocha \\ Caroline Pontes do Amaral \\ Horianna Cristina Silva de Mendonca}

Discentes do Curso de Fisioterapia da Universidade Federal de Pernambuco (UFPE), Brasil.

\section{Lívia Shirahige Gomes do Nascimento}

Mestranda do Programa de Pós Graduação em Fisioterapia da Universidade Federal de Pernambuco (UFPE), Brasil.

\section{Shirley Lima Campos}

Fisioterapeuta; Doutora em Engenharia Mecânica pela Universidade Federal de Minas Gerais (UFMG); Docente da Graduação e do Programa de Pós Graduação em Fisioterapia da Universidade Federal de Pernambuco (UFPE), Brasil.

\section{Maria das Graças Rodrigues de Araújo}

Fisioterapeuta; Doutora em Nutrição pela Universidade Federal de Pernambuco (UFPE), Brasil; Docente da Graduação e do Programa de Pós Graduação em Fisioterapia da Universidade Federal de Pernambuco (UFPE), Brasil.

E-mail: mgrodriguesaraujo@hotmail.com
RESUMO: Diabetes Mellitus, distúrbio metabólico crônico dos carboidratos com elevadas taxas de morbi-mortalidade, tem a neuropatia diabética periférica como complicação mais prevalente. $\mathrm{O}$ objetivo desse estudo foi verificar associação entre sensibilidade plantar e força muscular do tornozelo em pacientes diabéticos. Estudo transversal, com 18 pacientes, verificou-se que há associação entre sensibilidade plantar e força muscular do tornozelo nesses pacientes, avaliados quanto à sensibilidade tátil com monofilamentos (Semmes-Weisntein) em três pontos da região plantar nos dois pés, e força muscular de tornozelo, com teste de força manual, graduada de 0-5. Análise realizada por meio de experimentos SPSS 20.0, testes qui-quadrado e exato de Fisher $(p<0,05)$. Observou-se que $44,5 \%$ dos pacientes tinham perda da sensibilidade tátil; 38,8\% da dolorosa; $38,8 \%$ e 33,3\% diminuição da força dorso-flexora bilateral, porém a correlação dessas variáveis não foi estatisticamente significante. Portanto, não foi possível associar perda de sensibilidade plantar com diminuição da força muscular nesses pacientes diabéticos.

PALAVRAS-CHAVE: Diabetes Mellitus; Fisioterapia; Força Muscular; Neuropatia Diabética.

\section{ASSOCIATION BET WEEN MUSCLE FORCE AND FOOT SENSITIVENESS IN PATIENTS WITH DIABETES: A TRANSVERSAL STUDY}

\begin{abstract}
Diabetes mellitus, a chronic carbohydrate metabolic disorder with high morbo-mortality rates, also includes peripheral diabetic neuropathy as a predominant complication. Current analysis verifies the association between foot sensitiveness and muscle force of the ankle in patients with diabetes. A transversal study involving 18 patients revealed that there is an association between foot sensitiveness and muscle force at the ankle in patients who were evaluated with regard to tactile sensitiveness with monofilaments (Semmes-Weistein) at three sites of the sole the foot and the muscular force of the ankle, with tests for hand strength, graduated from 0 to 5 . Analysis SPSS 20.0, chi-square tests and Fisher's exact test $(p<0.05)$ were performed. Further, $44.5 \%$ of patients suffered loss of tactile sensitiveness; $38.8 \%$ had loss of pain sensitiveness; $38.8 \%$ and $33.3 \%$ had a decrease in the lateral dorsal-flexor force even though the co-relationship of variables was not statistically significant. Loss of sole sensitiveness could not be associated with decrease in muscle force in patients with diabetes.
\end{abstract}

KEY WORDS: Diabetes mellitus; Muscle Force; Diabetic Neuropathy; Physiotherapy. 


\section{INTRODUÇÃO}

Um grande problema de saúde pública mundial é o diabetes mellitus (DM), caracterizado como distúrbio metabólico crônico dos carboidratos com elevadas taxas de morbi-mortalidade (COELHO; SILVA; PADILHA, 2009). Segundo estimativas da Organização Mundial da Saúde (OMS), mais de 170 milhões de pessoas têm DM no mundo e este número será provavelmente maior que 0 dobro em 2030 (OMS, 2009; MENDES et al., 2011).

DM é caracterizado por hiperglicemia e glicosúria, resultante da produção ou utilização inadequada da insulina (RODRIGUES; SZYMANIAK; SOBRINHO, 2010). Suas principais formas são o diabetes tipo 1, quando existe deficiência absoluta de insulina, e o diabetes tipo 2, caracterizado por resistência à insulina com insuficiente elevação compensatória da secreção desse hormônio (FRÁGUAS; SOARES; BRONSTEIN, 2009).

De acordo com Sacco et al. (2007) e Amaral Júnior et al. (2014), a neuropatia diabética periférica (NP) é a complicação mais prevalente dessa doença e suas alterações são sensitivas (perda da sensibilidade dolorosa, percepção de pressão, temperatura e propriocepção); motoras (atrofia e enfraquecimento dos músculos do pé, resultando em deformidades); e autonômicas (diminuição ou ausência da secreção sudorípara com ressecamento da pele, rachaduras e fissuras).

Os estudos de Hirota et al. (2008) e os de Ochoa-Vigo et al. (2006) inferem que decorrente dos efeitos deletérios da $\mathrm{NP}$, associados às doenças vasculares periféricas (DVP) tem-se os transtornos tróficos da pele e da estrutura osteoarticular do pé culminando com as úlceras, o pé diabético, representando em $90 \%$ dos casos, uma das mais mutilantes complicações crônicas do DM devido ao aumento da suscetibilidade à infecção.

Dentre muitas alterações, a sensitiva antecede a disfunção motora devido à perda contínua dos axônios motores, causa subjacente da atrofia muscular, resultando em desnervação de fibras musculares com diminuição da força muscular, causando as alterações biomecânicas no pé (MENZ; LORD; STORGE; FITZPATRICK, 2004).

Já a DVP, que é caracterizada pela redução do fluxo sanguíneo, diminuição de nutrientes e oxigênio ao tecido e consequente dificuldade de cicatrização, favorece o surgimento de processos infecciosos, pelo fato de que condições isquêmicas reduzem a ação da antibioticoterapia (WU; DRIVER; WROBEL; ARMSTRONG, 2007).

Das características clínicas frequentes da DM, além das úlceras, tem-se a gangrena e amputações nos membros inferiores, resultando em pacientes com incapacidade funcional parcial ou definitiva (NEYRAARISMÉNDIZ; SOLÍS-VILLANUEVA; CASTILLO-SAYÁN; GARCÍA-RAMOS, 2012).

Essas complicações são advindas do mau controle da doença, com impacto social e econômico para as famílias, para o sistema de saúde e para a sociedade, tanto em países desenvolvidos como emergentes (ROCHA; ZANETTI; SANTOS, 2009).

O tratamento do DM consiste na manutenção dos níveis glicêmicos em taxas consideradas normais e as ações preventivas, de forma a postergar as complicações (TAVARES; CÔRTES; DIAS, 2010). Testes de avaliação de sensibilidade tátil, dolorosa e vibratória são descritos pela Sociedade Brasileira de Diabetes (2009) como ferramentas úteis para diagnóstico não-invasivo da neuropatia diabética e importantes na prevenção de pacientes com risco de desenvolverem o pé diabético (JIRKOVSKÁ; BOUCEK; WOSKOVÁ; BARTOS; SKIBOVÁ, 2001).

Portanto, torna-se imprescindível para a prática clínica da fisioterapia a identificação de informações sensoriais proveniente de receptores cutâneos plantares e a manutenção da força muscular nos diabéticos, de forma preventiva, a fim de preservar a funcionalidade e evitar quedas nesses indivíduos. Diante do exposto, o objetivo desse estudo foi verificar a associação entre a sensibilidade plantar e a força muscular do tornozelo em pacientes diabéticos.

\section{METODOLOGIA}

Trata-se de estudo transversal, com pacientes diabéticos oriundos de uma lista de atendimento do Núcleo de Apoio ao Idoso da Universidade Federal de Pernambuco (NAI-UFPE), em Recife. Este trabalho foi aprovado pelo CEP/CCS/UFPE - CAAE: 02990012.6.0000.5208. 
Um total de 18 (dezoito) voluntários foram avaliados no Laboratório de Cinesioterapia e Recursos Terapêuticos Manuais da UFPE no período de maio a julho de 2014, e selecionados de acordo com os seguintes critérios de inclusão: diagnóstico médico de diabetes mellitus, faixa etária superior a 40 anos, ambos os sexos e aprovação no Mini-Exame do Estado Mental (MEEM), versão brasileira (ponto de corte para indivíduos analfabetos de $18 \mathrm{e}$ alfabetizados de 25) segundo Lourenço e Veras (2006)
Foram excluídos os pacientes com alterações neurológicas, vasculares, musculoesqueléticas ou respiratórias que não estão relacionadas diretamente com a diabetes e/ou que se tornem fatores incapacitantes para a manutenção do equilíbrio e realização da marcha.

A avaliação da sensibilidade plantar foi realizada de acordo com o protocolo sugerido por Apelqvist et al. (2000), e os testes foram realizados pelo mesmo avaliador.

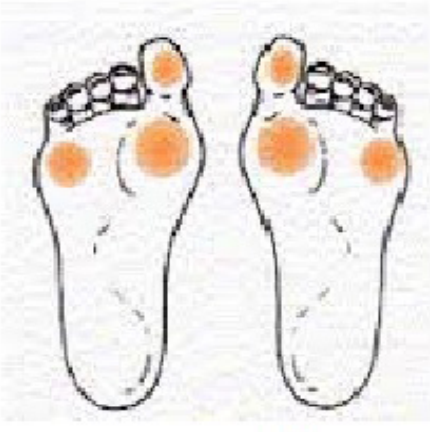

a

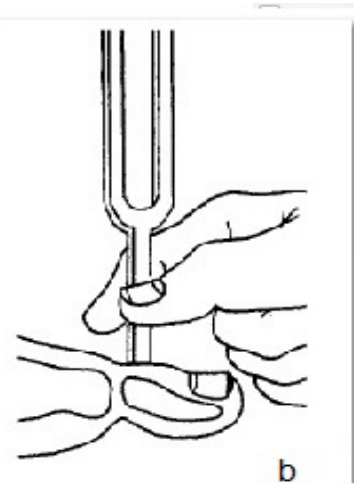

b

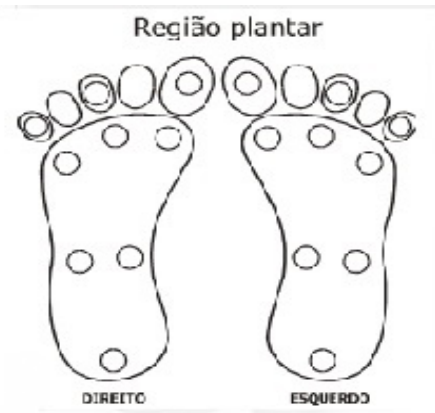

C

Figura 1. a, b, c

Fonte: Apelqvist et al. (2000)

A sensibilidade tátil foi avaliada usando $\mathrm{o}$ monofilamento de nylon de $10 \mathrm{~g}$ da marca SemmesWeisntein, em três pontos específicos nos pés dos pacientes (Figura 1a). Primeiramente foi aplicado o monofilamento na mão do paciente para demonstrar qual a sensação que ele poderia sentir, em seguida 0 paciente era posicionado sentado, com os pés relaxados sobre uma cadeira e orientados a manterem os olhos fechados. O filamento era aplicado perpendicularmente à fáscia plantar do pé, com uma força capaz de dobrálo e com duração de dois segundos, três vezes em cada ponto específico, em ordem aleatória. Após cada aplicação, perguntávamos se ele havia sentindo o contato do filamento e em qual local sentiu. Foram feitas três repetições, sendo duas reais e uma falsa, na qual o filamento não era pressionado, também aleatoriamente. A sensação de proteção era considerada presente em duas ou mais respostas certas e ausente em duas ou mais respostas incorretas.

Para avaliação da sensibilidade vibratória, usou-se o diapasão de $128 \mathrm{~Hz}$ com cursor. Inicialmente a aplicação era feita sobre o processo estilóide do rádio para prévio conhecimento do estímulo, em seguida o diapasão era pressionado na parte superior do hálux dos pés (Figura 1b), com o paciente de olhos fechados e alternando três aplicações "verdadeiras" e "falsas" (APELQVIST; BAKKER; VAN; NABUURS-FRANSSEN; SCHAPER, 2000).

De acordo com Apelquist et al. (2000), a sensibilidade dolorosa foi verificada em nove pontos plantares dos pacientes (Figura 1c), com a utilização de palitos descartáveis pontiagudos, aplicando-os paralelamente à face plantar do pé e sem penetrar na pele.

A força muscular foi avaliada segundo Kendall et al. (2007), correspondendo à escala de 0 a 5. De acordo com a força muscular, a escala pode ser graduada através do escore numérico, em ordem crescente: 0 (nula: ausência de contração); 1 (traço: há uma leve contração porém incapaz de produzir movimento); 2 (fraco: há movimento somente na ausência da gravidade); 3 (regular: consegue realizar movimento vencendo a gravidade); 4 (bom: consegue realizar movimento vencendo a gravidade e também uma resistência externa); 5 (normal: consegue realizar movimento superando 
uma resistência maior). Os testes eram realizados com o paciente em decúbito dorsal, com os pés fora da maca e o terapeuta demonstrava ao paciente os movimentos de flexão plantar, dorso-flexão, inversão e eversão, antes de executar o teste.

$\mathrm{Na}$ avaliação da regularidade glicêmica, os pacientes eram questionados sobre exames anteriores e se realizavam o monitoramento da glicose sanguínea. Aqueles que informavam que a glicemia se mantinha abaixo de 130 eram considerados regulares.

Os dados foram mostrados em média e desvio padrão para as variáveis quantitativas e em frequência para as variáveis categóricas. Para a análise estatística foi utilizado o programa SPSS 20.0 e foram realizados o teste qui-quadrado para comparação entre os grupos sensibilidade alterada e normal, e o teste exato de
Fisher para possíveis associações entre as variáveis de sensibilidade, força, idade, tempo de diagnóstico e regularidade da glicemia, adotando-se um nível de significância de $p<0,05$.

\section{RESULTADOS}

A amostra final foi composta por 18 indivíduos, sendo 15 mulheres (83,33\%) e 03 homens (16,66\%), com média de idade de $62,5 \pm 1,7$ anos, IMC de $31,33 \pm 1,33$ e tempo de diagnóstico de 9,33 \pm 1,62 anos (Figura 2). As características sociodemográficas e dados clínicos dos pacientes com e sem alteração de sensibilidade tátil se encontram na Tabela 1.

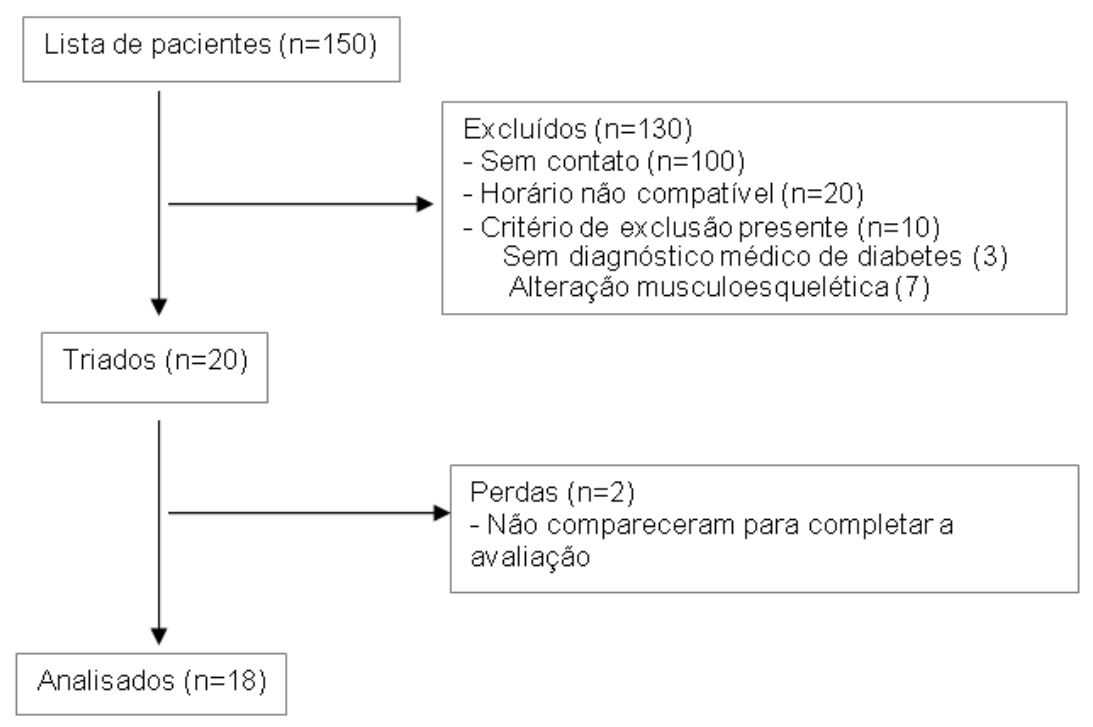

Figura 2. Fluxograma amostral do estudo 
Tabela 1. Dados Sociodemográficos e Clínicos dos Pacientes Diabéticos com Sensibilidade Tátil Alterada e Normal

\begin{tabular}{|c|c|c|c|}
\hline Características & Sensibilidade Alterada & Sensibilidade Normal & $p$ valor \\
\hline \multicolumn{4}{|l|}{ Sexo } \\
\hline Masculino, n (\%) & $3(100 \%)$ & 0 & 0,07 \\
\hline Feminino, n (\%) & $5(33,3 \%)$ & $10(66,6 \%)$ & \\
\hline Idade (anos), média ( $\pm \mathrm{DP})$ & $66,6 \pm 4,1$ & $59,3 \pm 7,7$ & 0,22 \\
\hline IMC, média ( $\pm \mathrm{DP})$ & $31,4 \pm 7,7$ & $31,23 \pm 7$ & 0,38 \\
\hline Tempo de diagnóstico (anos) média ( $\pm \mathrm{DP})$ & $11,5 \pm 8$ & $7,7 \pm 5,6$ & 0,68 \\
\hline \multicolumn{4}{|l|}{ Tipo de diabetes } \\
\hline Tipo I, n (\%) & 0 & $1(100 \%)$ & 1 \\
\hline Tipo II, n (\%) & $8(47 \%)$ & $9(53 \%)$ & \\
\hline \multicolumn{4}{|l|}{ Regularidade da glicemia } \\
\hline Regular & $5(50 \%)$ & $5(50 \%)$ & 0,66 \\
\hline Irregular & $3(37,5 \%)$ & $5(62,5 \%)$ & \\
\hline \multicolumn{4}{|l|}{ HAS } \\
\hline Presente & $5(45,45 \%)$ & $6(54,54 \%)$ & 1 \\
\hline Ausente & $3(42,85 \%)$ & $4(57,15 \%)$ & \\
\hline \multicolumn{4}{|l|}{ Umidade dos pés } \\
\hline Normal & $5(45,45 \%)$ & $6(54,54 \%)$ & 1 \\
\hline Seco & $3(42,85 \%)$ & $4(57,15 \%)$ & \\
\hline
\end{tabular}

Legenda: DP, desvio padrão; IMC, índice de massa corporal; HAS, hipertensão arterial sistêmica; $p$-valor para o teste qui-quadrado.

Em relação aos testes de sensibilidade, oito (44,5\%) pacientes demonstraram perda da sensibilidade tátil em um dos três pontos, sete $(38,8 \%)$ tiveram perda da sensibilidade dolorosa em pelo menos um dos noves pontos avaliados e apenas um (5,5\%) teve diminuição da sensibilidade vibratória no hálux direito (HD) (Tabela 2).

Tabela 2. Sensibilidade Plantar e Força de Dorso-Flexão de Tornozelo

\begin{tabular}{lr}
\hline & Frequência da amostra \\
\hline Sensibilidade tátil & \\
\hline Presente & $10(55,5 \%)$ \\
Ausente & $8(44,5 \%)$ \\
\hline Sensibilidade dolorosa & $11(61,2 \%)$ \\
\hline Presente & $7(38,8 \%)$ \\
Ausente & \\
\hline Força muscular & $11(61,2 \%)$ \\
\hline Dorsi-flexão D & $7(38,8 \%)$ \\
Normal (Grau 5) & \\
Diminuída (Grau 4) & $12(66,6 \%)$ \\
Dorsi-flexão E & $6(33,4 \%)$ \\
Normal (Grau 5) & \\
Diminuída (Grau 4)
\end{tabular}

Todos os pacientes tiveram uma boa força muscular do tornozelo, mas em 07 (38,8\%) houve diminuição da força na dorso-flexão à direita (DFD), 06 $(33,3 \%)$ na dorso-flexão à esquerda (DFE), 04 (22,2\%) na inversão esquerda (INVE) e direita (INVD), e apenas 01 com diminuição de força no movimento de flexão plantar direita (FLEPD). Força muscular na eversão (EVE) e flexão plantar esquerda (FLEPE) estavam preservados em todos os pacientes.

As diminuições de sensibilidade tátil e dolorosa se mostraram correlacionadas $(p=0,013)$, entretanto, não estão relacionadas com alterações da força muscular de DFD $(p=0,367 ; p=0,637)$ e de DFE $(p=0,638$; $p=1$ ) (Tabela 3). A idade mais avançada dos pacientes se correlacionou com as alterações de sensibilidade tátil $(p=0,02)$ e dolorosa $(p=0,005)$, o que não foi visto em relação ao tempo de diagnóstico $(p=0,145)-(p=1)$ e nem quanto a regularidade da glicemia $(p=0,367)-$ $(p=0,664)$. 
Tabela 3. Correlação entre as Variáveis de Sensibilidade e Força Muscular

\begin{tabular}{lcccc} 
& Sensibilidade Tátil & $\begin{array}{c}\text { Sensibilidade } \\
\text { Dolorosa }\end{array}$ & Dorso Flexão D & Dorso Flexão E \\
\hline Sensibilidade Tátil & $\mathbf{x}$ & $p=0,013^{*}$ & $p=0,36$ & $p=0,63$ \\
Sensibilidade Dolorosa & $p=0,013^{*}$ & $\mathbf{x}$ & $p=0,637$ & $p=1$ \\
Dorso Flexão D & $p=0,36$ & $p=0,637$ & $\mathrm{x}$ & $p<0,001^{*}$ \\
Dorso Flexão E & $p=0,63$ & $p=1$ & $p<0,001^{*}$ & $\mathrm{X}$ \\
\hline
\end{tabular}

$* p<0,05$ - Teste exato de Fisher

\section{DISCUSSÃO}

No presente estudo houve uma maior prevalência de pacientes diabéticos do tipo 2 (94,5\%) e de pacientes com HAS $(61,1 \%)$, o que corrobora o trabalho de Otero e colaboradores (2007), onde em um estudo com 54 pacientes, mostrou uma porcentagem de $96,3 \%$ de pacientes com diabetes tipo 2 e $61,1 \%$ com HAS.

Observou-se na amostra colhida nesse estudo, que oito pacientes tiveram perda da sensibilidade tátil e sete da sensibilidade dolorosa, uma tendência relativamente alta de pacientes com perda de sensibilidade protetora e consequente maior risco de ulcerações. Também foi visto no estudo de Ferreira e Torre (2013), que em uma população de 31 idosos diabéticos, 74,2\% destes apresentaram perda de sensibilidade tátil com 0 monofilamento de $10 \mathrm{~g}$.

Na pesquisa de Sacco et al. (2007) foi observado que o tibial anterior é um dos primeiros músculos a sofrer perda de força na neuropatia diabética; no presente estudo constatou-se uma força de grau 4 na dorsi-flexão direita em sete pacientes e na dorsi-flexão esquerda em seis. Andersen et al. (2004) avaliaram essa força muscular de tornozelo nos pacientes através do dinamômetro isocinético e encontraram diminuição dessa força de $14 \%$ para flexão plantar $(p=0,03)$ e de $17 \%$ para dorso flexão $(p=0,02)$ nos pacientes diabéticos quando comparados aos não diabéticos.

O presente estudo não obteve resultados significativos na relação da força muscular com a perda de sensibilidade, o que pode ser explicado pela forma como avaliamos a força muscular, manualmente, que de acordo com Andersen (2012), é um teste de fácil aplicabilidade e que não necessita de nenhum equipamento, mas nos fornece apenas informações semiquantitativas, enquanto que o dinamômetro isocinético é mais sensível em detectar fraquezas musculares. Eles correlacionaram a força muscular com a ausência e a presença de neuropatia diabética sintomática e assintomática, e mostraram que houve uma diminuição da força de dorso-flexão ( $\mathrm{r}=$. $0,35 ; p<0,05)$ e flexão plantar de $(\mathrm{r}=-0,47 ; p<0,005)$ em pacientes com neuropatia diabética sintomática em relação aos outros dois grupos.

Já nos resultados de Ijzerman et al. (2012), não houve diferença significativa na força muscular, avaliada através do dinamômetro isocinético, em relação aos pacientes com e sem neuropatia diabética. $\mathrm{O}$ estudo mostrou que esse resultado se deve à força muscular dos pacientes ser corrigida de acordo com o peso corporal e que no grupo controle os pacientes estavam mais aptos fisicamente.

Com relação à idade, nossos resultados mostram uma correlação de pacientes com idade mais elevada a apresentar tanto perda da sensibilidade tátil $(p=0,02)$, como dolorosa $(p=0,003)$, o que corrobora o estudo de Silva et al. (2013), que descreve a idade e o tempo de diagnóstico como fatores de risco para perda de sensibilidade protetora, em uma amostra de 56 pacientes diabéticos.

Os resultados do presente estudo são relevantes, mas algumas limitações devem ser consideradas como o tamanho da amostra e a falta de um grupo controle de pacientes não diabéticos para comparação de grupos distintos.

\section{CONCLUSÃO}

De acordo com nossos resultados, não foi possível associar a perda de sensibilidade plantar com a 
diminuição da força muscular em pacientes diabéticos. Considerando que o diagnóstico da neuropatia diabética envolve uma variedade de sinais e sintomas clínicos, testes simples e de fácil reprodutibilidade, e laboratoriais, tornam-se interessantes outros estudos que utilizem maior número amostral e avaliações mais sensíveis para detectar essa associação.

\section{AGRADECIMENTOS}

Nossos agradecimentos aos pacientes que participaram desse estudo e à Fundação de Amparo à Ciência e Tecnologia do Estado de Pernambuco (FACEPE) pelo financiamento desta pesquisa.

\section{REFERÊNCIAS}

AMARAL JÚNIOR, A. H.; AMARAL, L. A. H.; BASTOS, M. G.; NASCIMENTO, L. C.; ALVES, M. J. M.; ANDRADE, M. A. P. Prevencão de lesões de membros inferiores e redução da morbidade em pacientes diabéticos. Rev Bras. Ortop., v. 49, n. 5, p. 482-487, sep./oct. 2014.

ANDERSEN, H. Motor dysfunction in diabetes. Diabetes Metab Res Rev, v. 28, n. Suppl 1, p. 89-92, Fev. 2012.

ANDERSEN, H.; NIELSEN, S.; MOGENSEN, C. E.; JAKOBSEN, J. Muscle Strength in Type 2 Diabetes. Diabetes, v. 53, n. 6, p. 1543-1548, jun. 2004.

APELQVIST, J.; BAKKER, K.; VAN, H. W. H.; NABUURSFRANSSEN, M. H.; SCHAPER, N. C. International consensus and practical guidelines on the management and the prevention of the diabetic foot. Diabetes Metab Res Rev, v. 16, Suppl 1, p. S84-92, oct. 2000.

COELHO, M. S.; SILVA, D. M. G. V.; PADILHA, M. I. S. Representações sociais do pé diabético para pessoas com diabetes mellitus tipo 2. Rev Esc Enferm. USP, v. 43, n. 1, p. 65-71, 2009.

FERREIRA, L. G. F.; TORRE, M. V. Análise da Correlação entre Alterações Sensitivas e Mobilidade Funcional em Idosos Diabéticos, Teresina-PI. Rev Fisioter Saúde Funcional, v. 2, n. 1, p. 42-49, jan./jun. 2013.

FRÁGUAS, R.; SOARES, S. M. S. R.; BRONSTEIN, M. D. Depressão e diabetes mellitus. Rev Psiq Clín, v. 36, n. 3 , p. 93-99, 2009.

HIROTA, C. M. O.; HADDAD, M. C. L.; GUARIENTE, M. H. D. M. Pé diabético: o papel do enfermeiro no contexto das inovações terapêuticas. Ciênc Cuid e Saúde, v. 7, n. 1, p. 114-120, jan./mar. 2008.

IJZERMAN, T. H.; SCHAPER, N. C.; MELAI, T.; MEIJER, K.; WILLEMS, P. J.; SAVELBERG, H. Lower extremity muscle strength is reduced in people with type 2 diabetes, with and without polyneuropathy, and is associated with impaired mobility and reduced quality of life. Diabetes Res Clin Pract., v. 95, n. 3, p. 345-351, mar. 2012.

JIRKOVSKÁ, A.; BOUCEK, P.; WOSKOVÁ, V.; BARTOS, V.; SKIBOVÁ, J. Identification of patients at risk for diabetic foot. A comparison of standardized noninvasive testing with routine practice at community diabetes clinics. J Diabetes Complications, v. 15, n. 2, p. 63-68, march/ april. 2001.

KENDALL, F. P., MCCREARY, E. K.; PROVANCE, P. G. Músculos: provas e funções. 5. ed. São Paulo: Manole, 2007. ISBN13: 9788520424322.

LOURENÇO, R. A.; VERAS, R. P. Mini-exame do estado mental: características psicométricas em idosos ambulatoriais. Rev Saúde Pública, v. 40, n. 4, p. 712719, 2006.

MENDES, T. A. B.; GOLDBAUM, M.; SEGRI, N. J.; BARROS, M. B. A.; CESAR, C. L. G.; CARANDINA, L.; ALVES, M. C. G. P. Diabetes mellitus: fatores associados à prevalência em idosos, medidas e práticas de controle e uso dos serviços de saúde em São Paulo, Brasil. Cad. Saúde Pública, v. 27, n. 6, p. 1233-1243, jun. 2011.

MENZ, H. B.; LORD, S. R.; GEORGE, R. S.; FITZPATRICK, R. C. Walking stability and sensoriomotor function in older people with diabetic peripheral neuropathy. Phys Med Rehabil., v. 85, n. 2, p. 245-252, feb. 2004. 
NEYRA-ARISMÉNDIZ, L.; SOLÍS-VILLANUEVA, J.; CASTILLO-SAYÁN, Ó.; GARCÍA-RAMOS, F. Pie Diabético. Rev Soc Peru Med Interna, v. 25, n. 2, p. 76-88, 2012.

OCHOA-VIGO, K.; TORQUATO, M. T. C. G.; SILVÉRIO, I. A. S.; QUEIROZ, F. A.; DE-LA-TORRE-UGARTE-GUANILO, M. C.; PACE, A. E. Caracterização de pessoas com diabetes em unidades de atenção primária e secundária em relação a fatores desencadeantes do pé diabético. Acta Paul Enferm., v. 19, n. 3, p. 296-303, 2006.

ORGANIZAÇÃO MUNDIAL DE SAÚDE. Ação já contra o diabetes. 2009. Disponível em: <http://www.paho. org/bra/index.php?option $=$ com_docman\&task $=$ doc view\&gid $=322 \& I t e m i d=423>$ Acesso em: 18 ago. 2014.

OTERO, L. M.; ZANETTI, M. L.; TEIXEIRA, C. R. S. Características Sociodemográficas e Clínicas de Portadores de Diabetes em um Serviço de Atenção Básica à Saúde. Rev Latino-Am Enfermagem., v. 15, n. especial, set./ out. 2007.

PEDROA, H. Neuropatia diabética periférica. Sociedade Brasileira de Diabetes (SBD). 2009. Disponível em: $<$ http://www.diabetesebook.org.br/capitulo/neuropatiadiabetica-periferica > Acesso em: 22 ago. 2014.

ROCHA, R. M.; ZANETTI, M. L.; SANTOS, M. A. Comportamento e conhecimento: fundamentos para prevenção do pé diabético. Acta Paul. Enferm., v. 22, n. 1, p. 17-23, 2009.

RODRIGUES, A. N.; SZYMANIAK, N. P.; SOBRINHO, J. A. Influência das dermatoses na qualidade de vida do portador de diabetes mellitus. Ciênc. Saúde Coletiva, v. 15, Suppl 1, p. 1325-1332, 2010.

SACCO, I. C. N.; SARTOR, C. D.; GOMES, A. A.; JOÃO, S. M. A.; CRONFLI, R. Avaliação das perdas sensório-motoras do pé e tornozelo decorrentes da neuropatia diabética. Rev Bras Fisioter, v. 11, n. 1, p. 27-33, jan./fev. 2007.

SILVA, J. V.; SOUZA-MUÑOZ, R. L.; FIGUEREDO, A. S.; MELO, J. F. G.; FERNANDES, B. M. Fatores de Risco para Perda de Sensibilidade Plantar em Diabéticos: Estudo Caso-controle em Ambulatório de Endocrinologia. Rev Bras Ciênc Saúde, v. 17, n. 2, p. 113-120, 2013.
TAVARES, D. M. S.; CÔRTES, R. M.; DIAS, F. A. Qualidade de vida e comorbidades entre os idosos diabéticos. Rev Enferm UERJ, v. 18, n. 1, p. 97-103, jan./mar. 2010.

WU, S. C.; DRIVER, V. R.; WROBEL, J. S.; ARMSTRONG, D. G. Foot ulcers in the diabetic patient, prevention and treatment. Vasc Health Risk Manag, v. 3, n. 1, p. 65-76, feb. 2007.

Recebido em: 21 de setembro de 2015 Aceito em: 12 de novembro de 2015 